\title{
The uniqueness of the solution of the two-dimensional direct problem is the propagation of the action potential along the nerve fiber
}

\author{
A.J. Satybaev ${ }^{1 *}$, G.S. Kurmanalieva ${ }^{1,2}$ \\ ${ }^{1}$ Osh Technological University, Osh, Kyrgyzstan \\ ${ }^{2}$ Osh Technological University, Osh, Kyrgyzstan \\ *e-mail:abdu-satbaev@mail.ru
}

Key words: two-dimensional, parabolic, direct problem, hyperbolic, generalized, action potential, nerve fiber, axon, uniqueness of solution

Formulation of the problem: The two-dimensional problem of the process of propagation of the action potential along nerve fibers is modeled by the following problem of an equation of parabolic type [1]:

$C_{m}(x, y) v_{t}^{\prime}(x, y, t)=\frac{r_{a}(x, y)}{2 \rho_{a}(x, y)} \Delta v-\frac{v(x, y, t)}{\rho_{m}(x, y) \cdot l},(x, t) \in R_{+}^{2}, y \in R$,

$v(x, y, t)\left|\mathrm{t}<0 \equiv 0, \quad v_{x}^{\prime}(x, y, t)\right| x=0==h(y) \theta(t)+r(y) \theta_{1}(t)+p(y) \theta_{2}(t), t \in R_{+}, y \in R$,

where, $h(y), r(y), p(y)$ - are given functions, $\theta(t)$ - is the Heaviside theta function, $\theta_{1}(t)=t \theta(t), \theta_{2}(t)=\frac{t^{2}}{2} \theta(t), C_{m}-$ is the capacitance, $r_{a}-$ is the radius of the nerve fiber, $\rho_{a}, \rho_{m}$-are the resistivities of the nerve fiber plasma and the membrane material, respectively, $l-$ is the membrane thickness, $v(x, y, t)-$ is the intracellular action potential, $a$ and $m$ - indexes of nerve fibers and membranes, $\Delta v-$ is the Laplace operator.

Using the Laplace transform, the parabolic problem (1)-(2) is reduced to an equivalent problem of hyperbolic type [2].

For this hyperbolic problem, a theorem on the uniqueness of the solution is justified [3]. It is shown that the uniqueness of the parabolic problem is established from the equivalence of problems of hyperbolic and parabolic type.

\section{References}

1. Basharina O.V., Artyuhov V.G. (2007) Biophysics. Voronezh: VSU, 83 p.

2. Kabanikhin S.I. (2009) Inverse and ill-posed problems, Siberian Scientific Publishing. Novosibirsk. p. 458.

3. Satybae A.J., Anishchenko Yu.V. (2014) Uniqueness of the solution of the two-dimensional direct problem of geoelectrics with instantaneous and corded sources. Science and new technologies. 7:25-29. 